\title{
Concordancia entre la tomografía axial computarizada de abdomen, la ultrasonografía endoscópica y la punción por aspiración con aguja fina en el diagnóstico de lesiones sólidas sugestivas de neoplasias malignas de páncreas
}

\section{Diagnostic concordance of abdominal CT scans, Endoscopic Ultrasonography and Fine Needle Puncture Aspiration for solid pancreatic lesions suggestive of malignant neoplasms}

\author{
Raúl Antonio Cañadas Garrido, MD, ${ }^{\star}$ Rómulo Darío Vargas Rubio, MD, ${ }^{2}$ Oscar Mauricio Muñoz Velandia, MD, PhD, ${ }^{3}$ \\ Jenny Andrea Velásquez Merchán, MD, ${ }^{4}$ Michelle Sussan Behzadpour García. ${ }^{5}$
}

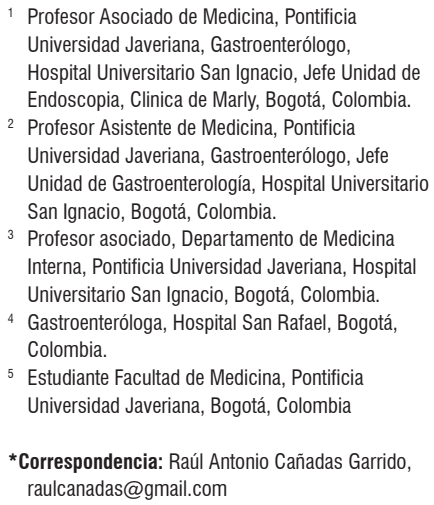

\begin{abstract}
Resumen
Introducción y objetivo: la realización de pruebas para el diagnóstico de lesiones sólidas de páncreas conduce frecuentemente a resultados discordantes, lo que genera confusión y retraso en las decisiones terapéuticas. La concordancia entre los resultados de la tomografía axial computarizada de abdomen con contraste (TAC), la ultrasonografía endoscópica (USE) biliopancreática y la punción por aspiración con aguja fina guiada por ultrasonografía endoscópica (PAAF-USE) no ha sido evaluada en nuestro medio. Materiales y métodos: se evaluó una serie de pacientes adultos con masas sólidas del páncreas sospechosas de malignidad, atendidos en el Hospital Universitario San Ignacio (HUSI) de Bogotá (Colombia), en los cuales se realizaron, por lo menos, dos de las siguientes pruebas: TAC, USE o PAAF-USE. Se evaluó la concordancia de los resultados, definidos como compatibles con neoplasia, no compatibles con neoplasia o resultado no conclusivo. Resultados: se incluyeron 57 pacientes. Un alto porcentaje de estos, con USE compatible con neoplasia, tuvieron resultados discordantes con la TAC $(33,3 \%)$ y con la PAAF-USE $(52,5 \%)$. La concordancia entre imágenes y PAAF-USE fue mínima (kappa = 0,02; intervalo de confianza [IC] $95 \%, 0,04-0,08$ ). La probabilidad de detectar un compromiso vascular fue significativamente mayor en la USE $(42,1 \%$ frente a $23,8 \%, p=0,06$ ) a diferencia del compromiso ganglionar, que fue detectado más frecuentemente por imágenes (TAC/resonancia magnética nuclear [RMN]) $(23,8 \%$ frente a $7,1 \%, p=0,01)$. Conclusiones: los resultados de este estudio sugieren un pobre acuerdo entre los diferentes métodos diagnósticos y advierten que es necesario implementar mejoras como la elastografía y medios de contraste, nuevas modalidades de aguja para la toma de muestras o la presencia de un citopatólogo in situ.
\end{abstract}

Palabras clave

Epidemiología, neoplasias pancreáticas, ultrasonografía biliopancreática, punción por aspiración con aguja fina (PAAF), estudios de concordancia.

\section{INTRODUCCIÓN}

El cáncer de páncreas es una neoplasia agresiva, de mal pronóstico, con una tasa de incidencia de 8-10 casos por
100000 habitantes al año. Representa el segundo tumor maligno gastrointestinal en frecuencia y es la cuarta causa de muerte por cáncer en adultos, con el agravante de que constituye una de las neoplasias malignas más difíciles de 


\begin{abstract}
Introduction and objective: Diagnostic tests for solid pancreatic lesions frequently produce discordant results which lead to confusion and delays of therapeutic decisions. Concordance among abdominal computed tomography with contrast, endoscopic ultrasound (EUS) pancreatobiliary and EUS guided fine needle aspiration had not previously been evaluated in Colombia. Materials and methods: We evaluated a series of adult patients with solid pancreatic masses suspected of malignancy treated at the San Ignacio University Hospital in Bogotá, Colombia. At least two of the following tests were performed: CT scans, EUS, and EUS guided fine needle aspiration. Results were defined as compatible with neoplasia, not compatible with neoplasia or inconclusive. Concordance of results was then evaluated. Results: Fifty-seven patients were included. A high percentage EUS results compatible with neoplasia were discordant with CT scan results and with EUS guided fine needle aspiration results ( $33.3 \%$ and $52.5 \%$, respectively). Agreement between imaging and EUS guided fine needle aspiration results was minimal (Kappa 0.02; 95\% Cl:-0.04 to 0.08). The probability of detecting vascular compromises was significantly higher for EUS (42.1\% vs. $23.8 \%$, p: 0.06$)$, but lymph node compromises were detected more frequently by imaging (CT or MRI) $(23.8 \%$ vs. $7.1 \%$, p: 0.01$)$. Conclusions: The results of this study suggest poor agreement between these diagnostic methods implying a need for improvements such as elastography and contrast media, new needle modalities for sampling, and/or the an on-site cytopathologist.
\end{abstract}

\title{
Keywords
}

Epidemiology; Pancreatic malignancies; Biliopancreatic ultrasound; PAAF; Concordance studies.

tratar, puesto que produce muy pocos síntomas en sus fases iniciales y habitualmente su diagnóstico es tardío, razón por la cual la opción de curación es casi nula (1).

A pesar de los múltiples avances de la última década, persisten pocas opciones para el manejo de la enfermedad en etapas avanzadas, con tasas de supervivencia a 5 años inferiores al $5 \%$ (2). Sin embargo, una proporción significativa de pacientes podrían extender su tiempo de supervivencia mediante cirugía, si estos tumores son diagnosticados en etapa temprana (3).

La detección de cánceres pancreáticos pequeños permite, sin duda, un mejor pronóstico que los cánceres grandes y es una estrategia necesaria para reducir las altas tasas de mortalidad. En este sentido, el papel de las técnicas de imagen (TAC, resonancia magnética) y la ultrasonografía endoscópica (USE) constituyen las mejores herramientas en el abordaje de estas lesiones y son, hoy en día, consideradas como indispensables para el adecuado enfoque de estudio de las lesiones sólidas del páncreas, cuando se trate de definir la malignidad o la benignidad.

La alta resolución de imagen de la USE es particularmente útil para detectar lesiones pancreáticas pequeñas que otras modalidades de pueden soslayar, en especial aquellas lesiones sólidas $<2 \mathrm{~cm}$. Por lo tanto, en la actualidad se considera que la USE es pieza fundamental y debería realizarse prácticamente en todos los pacientes con ictericia obstructiva de probable etiología neoplásica, en quienes la tomografía computarizada (TC) o la resonancia magnética (RM) no identifiquen una lesión pancreática evidente que explique la etiología. Además, la punción por aspiración con aguja fina guiada por (PAAF-USE) puede proporcionar muestras en estas lesiones de difícil identificación, a partir de métodos radiológicos, en el intento de obtener un diagnóstico histológico (4).

Ahora bien, la evaluación de las características operativas de las pruebas disponibles para el diagnóstico de las masas pancreáticas es un reto, si se considera que no existe un estándar de referencia definitivo. Normalmente, los investigadores han considerado como estándar de referencia un desenlace combinado que tiene en cuenta las muestras de tejido de los pacientes en los que se pudo realizar una extirpación completa del tumor, o la evolución clínica de los pacientes en los que no fue posible el manejo quirúrgico, sobre lo cual consideran que estos pacientes tienen una progresión tumoral y mortalidad precoces.

En la actualidad, se cuenta con varios métodos diagnósticos para la caracterización de lesiones sólidas neoplásicas de páncreas, sin que ninguno de ellos pueda considerarse como estándar de referencia único. La prueba de elección para el abordaje inicial suele ser una imagen radiológica, bien sea la tomografía axial computarizada (TAC) o la resonancia magnética nuclear (RMN) de abdomen. Se ha reportado que las imágenes (TAC/RMN) tienen una sensibilidad y una especificidad para el cáncer pancreático entre el 95 y el $96 \%$, respectivamente (5-8).

La reconstrucción multiplanar en la TAC es importante en la estadificación tumoral, dado que proporciona una visualización selectiva de importantes estructuras arteriales y venosas. Esto permite la visualización precisa de la relación del tumor primario con la arteria mesentérica superior 
(AMS), la vena mesentérica superior (VMS) y el tronco celíaco, lo cual contribuye a efectuar una evaluación de la invasión vascular y de la resecabilidad.

Entre tanto, la TAC es capaz de distinguir compresiones, invasión, estrechamiento u oclusión de la vena porta y de la VMS en la confluencia y permitir al cirujano determinar si una reconstrucción venosa es técnicamente factible. La precisión de la TC en la evaluación de invasión vascular no es fuerte, con estudios recientes que muestran una sensibilidad de solo el $60 \%$ y una especificidad del $94 \%$ (9). La TAC también desempeña un papel importante en la predicción de la irresecabilidad. Si el tumor rodea un vaso por más de $180^{\circ}$ grados y existe oclusión de la VMS o la vena porta, no habrá opciones quirúrgicas de reconstrucción. Recientemente, los estudios han demostrado que la sensibilidad de la TAC para la enfermedad irresecable está entre el 52 y el $91 \%$, y la especificidad oscila entre el 92 y el $100 \%$.

La RM ha demostrado ser igualmente sensible y específica en el diagnóstico y la estadificación del cáncer de páncreas como la TAC, con una sensibilidad combinada y una especificidad del 88 y $89 \%$, respectivamente. Por esta razón, la RM no es ampliamente utilizada como la modalidad de imagen primaria en la mayoría de los centros, a lo cual se suma su limitación por costos y disponibilidad.

Según los resultados de las imágenes radiológicas, frecuentemente se indica la realización de una USE biliopancreática con fines diagnósticos y el objetivo de mejorar la caracterización de las imágenes y la toma de muestras de la lesión. Así, el USE se realiza bajo sedación e implica un examen endoscópico del tracto gastrointestinal superior, mediante el uso del ecoendoscopio radial o lineal.

El transductor del ecoendoscopio se coloca en el duodeno o en el estómago, en proximidad directa con el páncreas, para que permita imágenes de alta resolución y detalladas de los vasos circundantes, los ganglios linfáticos y el lóbulo izquierdo del hígado, con el beneficio adicional de facilitar la realización de la punción por aspiración con aguja fina (PAAF) y obtener un diagnóstico citopatológico.

Como se mencionó, la USE es particularmente ideal para las lesiones $<2 \mathrm{~cm}$ o cuando existe una sospecha clínica de cáncer de páncreas, en la cual otras modalidades han fallado en identificar una masa y en obtener una confirmación histológica (Figuras 1A y 1B). En este contexto, la USE es considerada la técnica de imagen más sensible para la caracterización de los tumores pancreáticos, ya que alcanza valores reportados en la literatura del 89 al $100 \%$ para la detección del adenocarcinoma de páncreas (5-7).

A pesar de la alta sensibilidad de la USE, sigue siendo difícil diferenciar el adenocarcinoma del páncreas de otros tipos de lesiones sólidas, al considerar solo las características endosonográficas $(7,10)$. En este sentido, la utilización de la elastografía y los contrastes ha permitido la caracteri- zación de patrones que mejoran la sensibilidad visual de las imágenes, así como la orientación en la toma de la biopsia, para obtener el tejido y lograr la confirmación histológica de las lesiones, la cual es indispensable a la hora de decidir medidas terapéuticas.

La PAAF-USE es una técnica segura y útil en la obtención de material para el estudio citológico y, en algunos casos, para la toma de biopsias con obtención de tejido, lo cual permite el estudio histopatológico $(10,11)$. De igual forma, la PAAF-USE se ha convertido en el método estándar a fin de obtener muestras de tejido de lesiones pancreáticas asociadas, por fortuna, a un riesgo bajo de complicaciones y de siembras tumorales (12).

Los datos de metaanálisis sugieren que la sensibilidad y la especificidad en el diagnóstico de cáncer de páncreas es del $85-92 \%$ y del $96-98 \%$, respectivamente. Sin embargo, la PAAF- USE supone múltiples retos para la obtención de muestras representativas y suficientes a la hora del diagnóstico. Se requieren, entre otros elementos, el uso de una técnica adecuada, la experiencia del operador, el número de muestras (pases) y la presencia de un citopatólogo in situ (casi nunca disponible en los centros), sumados a las dificultades en la interpretación de los hallazgos histopatológicos durante la lectura efectuada por parte de los patólogos entrenados.

Como vemos, estas múltiples limitaciones condicionan que el rendimiento de la USE y la PAAF-USE no sea el mejor en algunos centros, y que la especificidad en muchas instituciones solo alcance el $88,6 \%$. Esta es especialmente limitada cuando no se dispone de un estudio citopatológico in situ (13).

Por su parte, los métodos radiológicos tampoco son infalibles y, en contraste, muestran un rendimiento muy pobre cuando la lesión es pequeña $(<2 \mathrm{~cm})$. Esto ocurre porque, dado su tamaño, las lesiones pueden no ser aparentes, lo cual tiene un impacto en las cifras de concordancia entre los exámenes radiológicos, la USE y la PAAF-USE.

En síntesis, la evaluación de las masas pancreáticas es especialmente difícil y constituye un reto diagnóstico, si se considera que no toda lesión sólida del páncreas es un adenocarcinoma. También es necesario comprender que el diagnóstico diferencial incluye neoplasias de relevancia clínica que suponen un enfoque distinto de tratamiento.

Entre estas condiciones se hallan el tumor carcinoide, el linfoma y las metástasis, todas ellas sumadas a la importancia de descartar lesiones benignas con apariencia de neoplasia como lesiones inflamatorias por pancreatitis crónica, el pseudotumor pancreático y la pancreatitis autoinmune con compromiso focal. La aparición de estas lesiones cambiaría totalmente el abordaje terapéutico, pues estas son de manejo no quirúrgico, razón por la cual se pueden evitar cirugías mayores, mórbidas e innecesarias.

En la práctica clínica, observamos con mucha frecuencia que son necesarias varias pruebas diagnósticas antes 

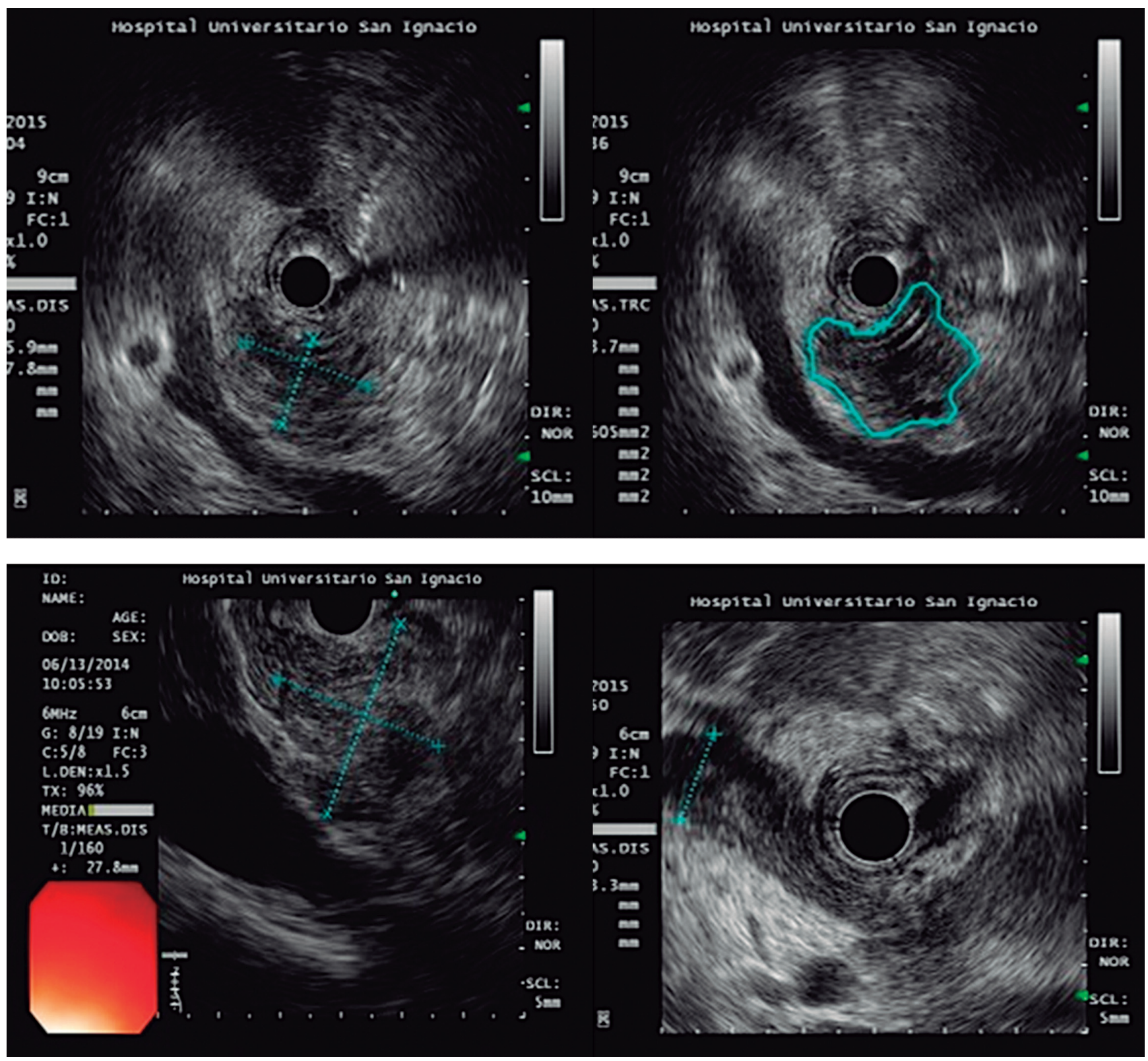

Figura 1. Imágenes de USE para el diagnóstico de lesiones sólidas sugestivas de neoplasias malignas de páncreas. A. Lesión neoplásica, hipoecoica en la cabeza del páncreas. B. Imagen hipoecoica irregular, sospechosa de neoplasia con PAAF negativa para malignidad. No toda masa sólida del páncreas es neoplasia maligna. Punción por aspiración con aguja fina (PAAF): adenocarcinoma de páncreas.

de llegar a una conclusión, lo que refleja la dificultad existente para establecer el diagnóstico definitivo de una lesión sólida del páncreas. Así, la realización de múltiples pruebas diagnósticas en estos pacientes conduce frecuentemente a que el personal médico se enfrente a resultados diferentes e incluso discordantes. Esto puede generar confusión para la toma de conductas terapéuticas y distorsionar la percepción del paciente frente la enfermedad. Además, puede contribuir a empeorar el pronóstico por el evidente retraso terapéutico, profundizado por las dificultades de nuestro sistema de salud.

Así pues, el objetivo del presente estudio es evaluar la concordancia entre las imágenes (TAC/RMN de abdomen), la USE y la PAAF-USE, con el fin de determinar si sus resultados pueden ser interpretados como clínicamente equivalentes en el diagnóstico de lesiones sólidas pan- 
creáticas sugestivas de adenocarcinoma, en un conjunto de pacientes manejados en el Hospital Universitario San Ignacio (HUSI).

\section{MATERIALES Y MÉTODOS}

Se realizó un estudio de pruebas diagnósticas a partir de la identificación de los pacientes con lesiones sólidas de páncreas, que fueron atendidos en el HUSI entre enero de 2014 y septiembre de 2017. Se incluyeron individuos mayores de edad con lesiones sólidas sugestivas de neoplasia, en los cuales estaban disponibles los resultados de, al menos, dos de las siguientes pruebas: una imagen radiológica (TAC/ RMN), USE biliopancreática o PAAF-USE.

Asimismo, se excluyeron los pacientes con imágenes de baja calidad que no fueran adecuadas para la lectura institucional. El estudio contó con la aprobación del Comité de Ética e Investigaciones de la institución. Los datos correspondientes a las características demográficas de los pacientes, así como los antecedentes médicos y la indicación de realizar un método diagnóstico, fueron obtenidos a partir de los registros consignados de forma sistemática en la base de datos de MEDICAP de la Unidad de Gastroenterología del HUSI y de la historia clínica electrónica.

Para realizar la TAC/RMN y la USE, se extrajo la información correspondiente a las características, la localización y el tamaño de la lesión; la presencia de un compromiso ganglionar, vascular o metastásico; la dilatación coledociana o del conducto pancreático; la existencia de una infiltración de grasa peripancreática, y la conclusión de la interpretación.

La TAC y la RMN fueron interpretadas por miembros del servicio de radiología, mientras que la USE, por un gastroenterólogo con entrenamiento formal en esta técnica. Entre tanto, un citopatólogo experto efectuó la evaluación de las muestras de PAAF-USE. Además, se registró si había presencia del patrón de panal de abejas, de una irregularidad de la membrana nuclear, de un nucléolo destacado en ausencia de inflamación y de un aclaramiento de la cromatina perinuclear.

Las variables continuas se expresaron como media, y la medida de dispersión como desviación estándar si siguieron una distribución normal; si no lo hicieron, se describieron como mediana y rango intercuartílico (RIC). Por su parte, las variables categóricas se expresaron como porcentajes.

Para establecer la concordancia entre las conclusiones de las imágenes, la USE y el reporte de la muestra tomada por PAAF-USE, se utilizó la aproximación de concordancia por consistencia, en la cual no se asume que alguna de las pruebas es el estándar de referencia (14). Este enfoque permite evaluar si los resultados de las diferentes pruebas son equivalentes entre ellas, pero no asume que los resultados de una u otra sean correctos. Por tanto, no permite determinar si alguna de las pruebas es mejor.

Se categorizaron las conclusiones de cada prueba como compatibles con neoplasia, no compatibles con neoplasia o resultado no conclusivo. Además, se analizó la concordancia de un compromiso ganglionar y vascular, mediante TAC, RMN o USE y se describió si estaba o no presente dicho compromiso.

A fin de realizar la evaluación, se utilizó un estadístico kappa no ponderado y se fijó un nivel de significación alfa (a) de 0,05. Los cálculos fueron desarrollados utilizando el programa estadístico denominado Stata (15).

\section{RESULTADOS}

Se identificaron 137 pacientes con masas pancreáticas sospechosas de malignidad, de los cuales 57 cumplieron los criterios de inclusión. Las características demográficas de la población se presentan en la Tabla 1. La mediana de edad fue de 64 años (RIC = 42-83), mientras que la indicación clínica más frecuente para el inicio de los estudios fueron ictericia $(37 \%)$ y dolor abdominal (35\%). La comorbilidad más común fue la pancreatitis crónica (64\%). Además, la totalidad de los pacientes incluidos en el estudio contaban con una USE biliopancreática: 42 (74\%) tenían disponibles las imágenes radiológicas diagnósticas y 40 (70\%) contaban con un reporte de patología con muestras aptas para la lectura. Es necesario destacar que 25 pacientes (44\%) tenían disponibles las tres pruebas diagnósticas de forma simultánea (Figura 2).

Tabla 1. Características demográficas de los pacientes incluidos

\begin{tabular}{lcc}
\hline \multicolumn{1}{c}{ Variable } & \multicolumn{2}{c}{$\mathrm{n=57}$} \\
\hline Edad en años, mediana (RIC) & \multicolumn{2}{c}{$64(42-83)$} \\
Sexo, $\mathrm{n}(\%)$ & $\mathrm{n}$ & $\%$ \\
$\quad$ Masculino & 26 & 45,6 \\
Estado civil & & \\
$\quad$ Unión libre & 2 & 3,5 \\
$\quad$ Casado & 27 & 47,3 \\
$\quad$ Soltero & 13 & 22,8 \\
$\quad$ Otro & 15 & 26,3 \\
Antecedentes patológicos & & \\
$\quad$ Pancreatitis crónica & 35 & 61,4 \\
$\quad$ Diabetes mellitus & 12 & 21 \\
$\quad$ Ninguno & 8 & 14 \\
Tabaquismo* & & \\
$\quad$ Sí & & \\
\hline
\end{tabular}

RIC: rango intercuartílico; *tabaquismo definido como el consumo activo de tabaco al momento del diagnóstico. 


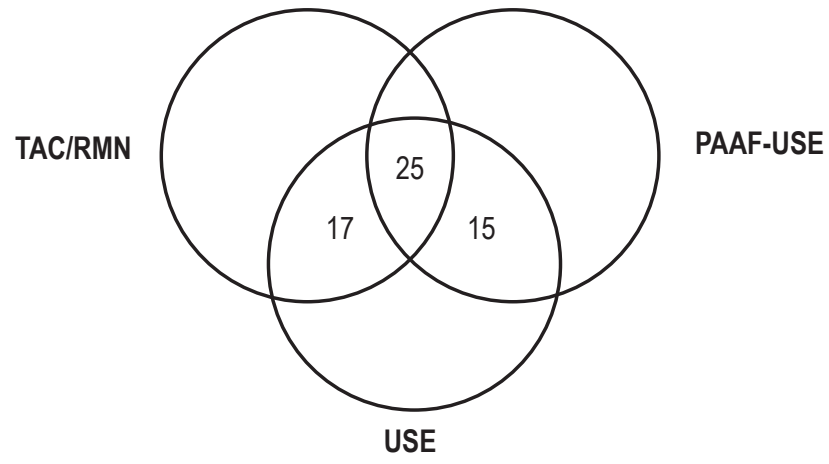

Figura 2. Disponibilidad de las pruebas diagnósticas para los pacientes incluidos. TAC: tomografía axial computarizada; RMN: resonancia magnética nuclear; PAAF: puncion por aspiración con aguja fina guiada; USE: ultrasonografía endoscópica

De acuerdo con los hallazgos reportados en la USE, la mayoría de las lesiones tenían un tamaño $>3 \mathrm{~cm}$ (59\%), con una mayor frecuencia de localización en la cabeza del páncreas (93\%). Un porcentaje significativo de los pacientes tuvieron un compromiso del colédoco y del conducto pancreático de forma simultánea, tanto en la USE como en la imagen radiológica ( 38 y $45 \%$, respectivamente).

La USE identificó la existencia de masas en 10 casos que no fueron detectados por imágenes radiológicas. La mayoría de estas lesiones estuvieron ubicadas en la cabeza del páncreas. La probabilidad de encontrar un compromiso vascular fue significativamente más alta en la USE (42,1\% frente al $23,8 \%, p=0,06$ ), a diferencia del compromiso ganglionar, el cual fue detectado en una proporción más alta de pacientes mediante las imágenes (TAC/RMN) $(23,8 \%$ frente al $7 \%, p=0,01)$. A continuación, se presentan las características de las lesiones encontradas por la TAC y la USE biliopancreática (Tabla 2).

De las 40 muestras aptas para lectura, solo el 47,5\% ( $\mathrm{n}=19)$ fueron compatibles con una lesión neoplásica. La característica más frecuentemente encontrada en las lecturas de la PAAF fue el aclaramiento de la cromatina (57,5\%). A continuación, se describen los hallazgos histológicos de las muestras tomadas por PAAF-USE (Tabla 3).

De acuerdo con los resultados de la USE, todos los pacientes $(\mathrm{n}=57)$ fueron compatibles con neoplasia del páncreas, a diferencia de lo reportado en las imágenes y en la PAAF, en las cuales solo el 66,7 \% y el 47,5\% fueron compatibles, respectivamente. A continuación, se presentan las conclusiones de las diferentes pruebas diagnósticas, categorizadas como compatibles con neoplasia del páncreas, no compatibles con neoplasia o resultado no conclusivo (Tabla 4).

El coeficiente kappa de concordancia entre la USE y las imágenes para clasificar las lesiones según las tres categorías
Tabla 2. Características de las lesiones encontradas por imágenes o por USE

\begin{tabular}{|c|c|c|}
\hline Variables & $\begin{array}{c}\text { Ultrasonografía } \\
\text { endoscópica } \\
n=57\end{array}$ & $\begin{array}{c}\text { Imagen } \\
\text { (TAC/RMN) } \\
n=42\end{array}$ \\
\hline \multicolumn{3}{|l|}{ Localización de la lesión } \\
\hline Cabeza & $53(93 \%)$ & $30(71 \%)$ \\
\hline Cuerpo & $4(7 \%)$ & $2(4,7 \%)$ \\
\hline Cola & 0 & 0 \\
\hline Ausencia de lesión & 0 & $10(23,8 \%)$ \\
\hline \multicolumn{3}{|l|}{ Tamaño de la lesión } \\
\hline $0-2 \mathrm{~cm}$ & $10(17,5 \%)$ & $6(14 \%)$ \\
\hline $2-3 \mathrm{~cm}$ & $15(26,3 \%)$ & $4(7,32 \%)$ \\
\hline$>3 \mathrm{~cm}$ & $32(56,2 \%)$ & $22(53,6 \%)$ \\
\hline Ausencia de lesión & 0 & $10(24,3 \%)$ \\
\hline \multicolumn{3}{|l|}{ Compromiso ductal } \\
\hline Dilatación del colédoco & $13(22 \%)$ & $11(26 \%)$ \\
\hline $\begin{array}{l}\text { Dilatación del conducto } \\
\text { pancreático }\end{array}$ & $5(8,7 \%)$ & $5(11 \%)$ \\
\hline $\begin{array}{l}\text { Dilatación del pancreático y del } \\
\text { colédoco }\end{array}$ & $22(38 \%)$ & $19(45 \%)$ \\
\hline Sin dilatación & $17(29,8 \%)$ & $7(16 \%)$ \\
\hline \multicolumn{3}{|l|}{ Compromiso vascular } \\
\hline Sí & $24(42,1 \%)$ & $10(23,8 \%)$ \\
\hline No & $33(57,8 \%)$ & $32(76,2 \%)$ \\
\hline \multicolumn{3}{|l|}{ Compromiso ganglionar } \\
\hline Sí & $4(7,0 \%)$ & $10(23,8 \%)$ \\
\hline No & $53(92,9 \%)$ & $32(76,2 \%)$ \\
\hline \multicolumn{3}{|l|}{ Infiltración grasa } \\
\hline Sí & $0(0 \%)$ & $4(9,3 \%)$ \\
\hline No & $57(100 \%)$ & $38(90,4 \%)$ \\
\hline
\end{tabular}

TAC: tomografía axial computarizada; RMN: resonancia magnética nuclear

ya descritas no fue evaluable, dado que en todas las USE las lesiones pancreáticas fueron interpretadas como compatibles con neoplasia. De los 42 pacientes en los que se disponía de ambas imágenes, la TAC o la RMN, se reportó que 28 eran compatibles, mientras que 3 fueron no compatibles con neoplasia y 11 tuvieron resultados no conclusivos.

Asimismo, 40 pacientes disponían simultáneamente de la USE y de la biopsia tomada por PAAF. Las pruebas fueron interpretadas en la USE como lesiones pancreáticas compatibles con neoplasia. De ellas, 19 (47,5\%) tuvieron el mismo hallazgo en la PAAF, mientras que 15 no fueron compatibles con neoplasia y 6 no fueron conclusivas (Tabla 4).

Entre tanto, la Tabla 5 muestra la evaluación de concordancia entre los resultados de las imágenes radiológicas 
Tabla 3. Hallazgos histológicos de las muestras tomadas por PAAF-USE

\begin{tabular}{ll}
\hline \multicolumn{1}{c}{ Variables } & \multicolumn{1}{c}{$\mathbf{n = 4 0}$} \\
\hline Patrón de panal de abejas irregular & \\
Sí & $3(7,5 \%)$ \\
No & $37(92,5 \%)$ \\
Aumento del tamaño del núcleo & \\
Sí & $19(47,5 \%)$ \\
No & $21(52,5 \%)$ \\
Irregularidad y reforzamiento de la membrana nuclear & \\
Sí & $17(42,5 \%)$ \\
No & $23(57,5 \%)$ \\
Núcleo destacado en ausencia de inflamación & \\
Sí & $4(10 \%)$ \\
No & $36(90 \%)$ \\
Aclaramiento de la cromatina & \\
Sí & $23(57,5 \%)$ \\
No & $17(42,5 \%)$ \\
\hline
\end{tabular}

PAAF: punción por aspiración con aguja fina; USE: ultrasonografía endoscópica

Tabla 4. Clasificación de las lesiones según el resultado de las imágenes, la USE y la PAAF

\begin{tabular}{lccc}
\hline \multicolumn{1}{c}{ Variables } & $\begin{array}{c}\text { USE } \\
(\mathbf{n}=\mathbf{5 7})\end{array}$ & $\begin{array}{c}\text { Imagen } \\
(\text { TAC/RMN) } \\
(\mathbf{n}=\mathbf{4 2})\end{array}$ & $\begin{array}{c}\text { PAAF } \\
(\mathbf{n}=\mathbf{4 0})\end{array}$ \\
\hline $\begin{array}{l}\text { Compatibles con neoplasia } \\
\text { de páncreas }\end{array}$ & $57(100 \%)$ & $28(66,7 \%)$ & $19(47,5 \%)$ \\
$\begin{array}{l}\text { No compatibles con } \\
\text { neoplasia de páncreas }\end{array}$ & 0 & $3(7,1 \%)$ & $15(37,5 \%)$ \\
No diagnóstico & 0 & $11(26,2 \%)$ & $6(15,0 \%)$ \\
\hline
\end{tabular}

PAAF: punción por aspiración con aguja fina; USE: ultrasonografía endoscópica; RMN: resonancia magnética nuclear y de la biopsia PAAF-USE, sobre 25 pacientes en los que se tuvieron disponibles los resultados de las dos pruebas diagnósticas. El coeficiente kappa fue de 0,02 (intervalo de confianza [IC], $95 \%$ : -0.04-0,08); 14 casos tuvieron resultados discordantes.

En cuanto al compromiso vascular, la concordancia entre imágenes y la USE fue moderada (kappa $=0,4240$; IC, $95 \%$ : 0,3570-0,4920), mientras que para el compromiso ganglionar fue baja (kappa $=0,2199$; IC, $95 \%$ : 0,15990,2799).

\section{DISCUSIÓN}

Los resultados de este estudio sugieren la existencia de un pobre acuerdo entre los diferentes métodos utilizados para el diagnóstico de lesiones del páncreas sugestivas de malignidad. A pesar de que todos los pacientes incluidos en nuestro estudio presentaron lesiones observadas en la USE sugestiva de neoplasia, solo en el 47,5\% de las muestras tomadas por PAAF se documentó una neoplasia, y en el $66,7 \%$ de los casos se obtuvieron hallazgos imagenológicos compatibles. De forma similar, la concordancia entre los hallazgos de las muestras tomadas por PAAF y las imágenes diagnósticas fue mínima.

Es importante resaltar que la mayoría de nuestros pacientes tenían lesiones con un tamaño $>2 \mathrm{~cm}$ en el momento del diagnóstico y que dichas lesiones ya presentaban un compromiso metastásico $>3 \mathrm{~cm}$, al igual que dilatación en el conducto pancreático y el colédoco. Otros autores han reportado hallazgos similares, lo cual sugiere que la mayoría de los casos se detectan en estadios avanzados (14).

Este es el primer estudio que evalúa la concordancia entre los tres métodos más aceptados actualmente en nuestro medio para la evaluación de las lesiones sólidas de páncreas en una institución colombiana. Para ello, clasifica-

Tabla 5. Concordancia entre los resultados de las imágenes y el resultado de la biopsia PAAF para el diagnóstico de masas de páncreas

\begin{tabular}{|c|c|c|c|c|c|c|}
\hline & & & \multicolumn{3}{|c|}{ Imágenes* } & \multirow[t]{2}{*}{ Total } \\
\hline & & & Compatible & No compatible & No conclusivo & \\
\hline \multirow[t]{6}{*}{ Biopsia PAAF } & Compatible & Recuento & 9 & 6 & 4 & 19 \\
\hline & & $\%$ del total & 36 & 24 & 16 & 76 \\
\hline & No compatible & Recuento & 1 & 2 & 0 & 3 \\
\hline & & $\%$ del total & 4 & 8 & 0 & 12 \\
\hline & No conclusivo & Recuento & 2 & 1 & 0 & 3 \\
\hline & & $\%$ del total & 8 & 4 & 0 & 12 \\
\hline \multirow[t]{2}{*}{ Total } & & Recuento & 12 & 9 & 4 & 25 \\
\hline & & $\%$ del total & 48 & 36 & 16 & 100 \\
\hline
\end{tabular}

PAAF: punción por aspiración con aguja fina; *imágenes entendidas como tomografía axial computarizada de abdomen o resonancia magnética de abdomen. 
mos las imágenes de endosonografía biliopancreática como lesiones sólidas y sugestivas de adenocarcinoma de páncreas, de acuerdo con los criterios establecidos en la USE por Gonçalves y colaboradores (15) y en imagen (TAC/ RMN) por Low y colaboradores (16).

Contrario a lo esperado, los hallazgos de la localización no fueron concordantes en un alto porcentaje de casos (kappa $=0,03$ ). Esto puede ser secundario a la presencia de lesiones $<1 \mathrm{~cm}$, las cuales no son claramente detectables mediante TAC o RMN, tal y como lo demostraron los estudios realizados por Sakamoto y colaboradores (3). Destacamos que 10 pacientes no tenían identificadas las lesiones sólidas por imágenes, las cuales fueron ubicadas y caracterizadas con la USE. Esto muestra el alto rendimiento de la prueba cuando se trata de detectar de lesiones pequeñas $<2 \mathrm{~cm}$.

En un reciente metaanálisis, la TC mostró una menor sensibilidad que la USE en la estadificación ganglionar (24\% frente a $58 \%$ ) y la invasión vascular ( $58 \%$ frente a $86 \%$ ). Sin embargo, las especificidades para la estadificación ganglionar ( $88 \%$ frente a $85 \%$ ) y la invasión vascular (95\% frente a $93 \%$ ) fueron comparables en estudios en los que se realizaron ambas técnicas de imagen.

Los resultados obtenidos en nuestro estudio demuestran que la escanografía y la RMN tienen la capacidad de identificar la infiltración de la grasa peripancreática y el compromiso nodular en una mayor proporción de casos. Esto sugiere que, aunque la concordancia entre las imágenes (TAC/RMN) y la USE es baja, puede justificarse la realización de estos estudios, dado que ofrecen información complementaria.

Así, nuestros hallazgos sugieren que un alto porcentaje de pacientes con USE compatible con neoplasia no presentan resultados histológicos compatibles con dicho diagnóstico. Esto puede ser secundario a varios factores como la cantidad y la calidad de la muestra, la ausencia de un patólogo en la sala para la verificación del contenido celular adecuado en el análisis o la alta pendiente en la curva de aprendizaje para la interpretación de la citología, lo cual fue advertido por Holt y colaboradores (14).

La PAAF- USE tiene buenos resultados y es la técnica de imagen preferida para guiar la toma de la muestra. Sin embargo, una gran cantidad de factores influyen en el rendimiento diagnóstico de la citología de las lesiones pancreáticas, entre ellos, el tamaño de la aguja, la obtención de bloques de tejido frente a laminillas y el uso de una evaluación rápida en el sitio (Rapid On-Site Evaluation, ROSE) para el análisis preliminar de la muestra; se debe garantizar que esta sea representativa.

Todos estos factores aún permanecen sin estandarizar y, básicamente, dependen de las preferencias locales, la viabilidad o la factibilidad. Parece claro entonces que esta falta de homogeneidad puede explicar, al menos de forma parcial, la gran variabilidad en el rendimiento diagnóstico de la PAAF de páncreas reportada en la literatura, el cual tiende a ser $<80 \%$.

También parece claro que el uso de agujas grandes (19 G) que producen muestras de tejido mayores, así como la ROSE del material por parte de citopatólogos expertos, aumentan significativamente el rendimiento diagnóstico de la PAAF. No obstante, la utilización de esta prueba sigue siendo frustrante en la práctica clínica diaria en muchos centros del mundo (17).

A pesar de que nuestros resultados estuvieron por debajo de lo esperado, no son sorprendentes, en vista de que demuestran la realidad de muchos centros que inician el camino a la excelencia en el estudio de las enfermedades biliopancreáticas. Para la toma de la PAAF en nuestros pacientes, utilizamos agujas de 19, 22 y 25 G, con una técnica de succión con liberación de la presión negativa antes de retirar la aguja del objetivo, y también efectuamos la técnica de retirar lentamente el estilete (pull), tal y como ha sido recomendado por las guías de la Sociedad Europea de Endoscopia Gastrointestinal (European Society for Gynaecological Endoscopy, ESGE) para el estudio de masas sólidas del páncreas (18).

Resaltamos la importancia de la larga curva de aprendizaje en la USE y en el acercamiento y la utilización de las nuevas agujas disponibles para la toma de muestras del páncreas por ultrasonido. También destacamos la necesidad de realizar un estudio interdisciplinario que permita una amplia comunicación con los diferentes grupos (gastroenterología, patología, radiología, cirugía, entre otros) cuando se abordan las lesiones sólidas del páncreas.

De todos estos aspectos, quizás el que mayor impulso ha tenido es el desarrollo de las diferentes agujas y de los métodos complementarios en imágenes por ultrasonido. En este sentido, las agujas se desarrollaron con un diseño de bisel inverso (ProCore, Cook Medical, Bloomington, Indiana), lo cual ha derivado en una mejor evaluación histológica $(81,1 \%$ frente a $69,4 \%, p=0,048)$ o en un número menor de pases necesarios para el diagnóstico que las agujas estándar de PAAF.

Entre tanto, las agujas con un diseño de punta Franseen (Adquirir, Boston Scientific Corp, Natick, Massachusetts, Estados Unidos) y aquellas con un diseño de punta de horquilla (SharkCore, Medtronic, Minneapolis, Minesota, Estados Unidos) pueden proporcionar muestras más grandes que la aguja estándar. En efecto, el rendimiento de ambas es comparable con el desempeño observado a partir de la histología de tejido.

De otro lado, la USE convencional sufre limitaciones en el diagnóstico de las lesiones de páncreas, dado que la mayoría de las lesiones sólidas se manifiestan como masas hipoecoicas, lo cual complejiza diferenciar entre las masas benignas y las malignas. La evaluación de la vasculari- 
zación es una forma de mejorar la caracterización de las lesiones digestivas y esto puede lograrse mediante el uso de la técnica desarrollada recientemente: la USE con contraste armónico (Contrast-enhanced Harmonic Endoscopic Ultrasound, CH-EUS).

Con el progreso reciente de la $\mathrm{CH}$-EUS, es probable que esta prueba desempeñe un papel más importante en el diagnóstico diferencial de las lesiones biliopancreáticas. De hecho, se estima que en el futuro la CH-EUS pueda convertirse en una herramienta alternativa útil para la evaluación de patologías que involucran la angiogénesis tumoral (19).

Entre tanto, la precisión global de la PAAF en el diagnóstico del adenocarcinoma pancreático es, aproximadamente, del $85 \%$. El uso de agentes de contraste durante la USE, con objeto de resaltar la vascularización y las partes necróticas de las masas pancreáticas, puede mejorar la orientación de la biopsia (20).

Nuestros hallazgos implican que no se debería asumir el resultado de la patología como método de referencia para hacer el diagnóstico de adenocarcinoma de páncreas, dado que puede existir un porcentaje significativo de falsos negativos, tal y como se demuestra en la literatura mundial. Dicho de otra forma, asumir el resultado de la patología como método de referencia puede generar falsos resultados y contribuir al diagnóstico tardío en un porcentaje significativo de casos, lo cual permite la progresión de la enfermedad y puede hacer que los pacientes no sean quirúrgicamente tratables.

Así, el método de referencia para la evaluación de las pruebas diagnósticas en el estudio de las masas pancreáticas deberá considerar no solo el reporte de la patología, sino también involucrar la epidemiología, la clínica, la evolución de los pacientes, su progresión y la suma complementaria de los métodos diagnósticos.

Vale la pena recalcar que el uso únicamente de la tomografía sin ultrasonografía y biopsia tiene una alta probabilidad de pérdida de información complementaria, la cual permite definir la conducta terapéutica. Por tanto, se considera que no es posible elaborar una estrategia terapéutica basada solamente en los resultados de la imagen (TAC/RMN).

Nuestro estudio tiene limitaciones que deben considerarse. La primera de ellas es que no todos los pacientes tuvieron disponibles las tres pruebas diagnósticas. Esto refleja lo que sucede en la vida real durante el manejo y el diagnóstico de estos pacientes en quienes las limitaciones del sistema de salud o la oportunidad de acceso al método diagnóstico no permiten un seguimiento adecuado. Decidimos, entonces, incluir el análisis de la comparación de los resultados de cada par de pruebas diagnósticas, considerando que una mayor cantidad de información mejora la precisión de la estimación de concordancia entre estas.
A pesar de las limitaciones, los datos sugieren que los resultados de imagen (TAC/RMN), la USE y la PAAFUSE no pueden ser interpretados como clínicamente equivalentes $y$, por lo tanto, no se puede asumir que una sola de estas pruebas orienta a determinar conductas terapéuticas, excepto en casos excepcionales. Será necesaria la realización de pruebas combinadas para efectuar una valoración más confiable de los pacientes con este tipo de lesiones. Además, se deben desarrollar otros estudios que comparen las diferentes pruebas diagnósticas con un estándar de referencia que incluya el seguimiento clínico a través del tiempo de los pacientes.

Así pues, los resultados de este estudio sugieren que existe un espacio para implementar nuevas herramientas que mejoren la sensibilidad diagnóstica de la USE, como la elastografía y los medios de contraste, además de incorporar diferentes modalidades de agujas para la obtención de tejidos que contribuyan a mejorar el rendimiento de la toma de biopsia. Todo ello, unido a la necesidad de la presencia de un citopatólogo in situ, a fin de realizar un análisis inmediato de las muestras obtenidas que contengan material representativo.

La disponibilidad de dicho recurso resulta rentable, si se considera que se logra la misma precisión del $87 \%$ con solo 2,1 pases, en comparación con los 4 pases necesarios cuando la evaluación en tiempo real de las muestras no estuvo disponible.

\section{CONCLUSIONES}

Los resultados de este estudio siguieren que existe un pobre acuerdo entre los diferentes métodos diagnósticos en las lesiones sólidas de páncreas. Asimismo, los datos obtenidos conducen a la necesidad de implementar mejoras en el diagnóstico de estas lesiones que, aun con la biopsia, son de difícil interpretación, lo que genera incertidumbre terapéutica. Sin embargo, la decisión quirúrgica no debería ser retardada si hay una alta sospecha clínica de cáncer de páncreas, a pesar de que la definición de alta sospecha clínica de cáncer de páncreas es aún poco clara.

\section{Responsabilidades éticas}

Los autores declaran que para esta investigación no se han realizado experimentos en seres humanos ni en animales.

\section{Confidencialidad de los datos}

Los autores declaran que en este artículo no aparecen datos de pacientes. 


\section{Conflicto de intereses}

Los autores declaran no tener conflictos de interés.

\section{REFERENCIAS}

1. Jemal A, Siegel R, Ward E, Hao Y, Xu J, Thun MJ. Cancer statistics, 2009. CA Cancer J Clin. 2009;59(4):225-49. http:// dx.doi.org/10.3322/caac.20006

2. Kleeff J, Michalski C, Friess H, Büchler MW. Pancreatic cancer: frombench to 5-year survival. Pancreas. 2006;33(2):1118. https://doi.org/10.1097/01.mpa.0000229010.62538.f2

3. Helmstaedter L, Riemann JF. Pancreatic cancer--EUS and early diagnosis. Langenbecks Arch Surg. 2008;393(6):9237. https://doi.org/10.1007/s00423-007-0275-1

4. Yoshida T, Yamashita Y, Kitano M. Endoscopic Ultrasound for Early Diagnosis of Pancreatic Cancer. Diagnostics (Basel). 2019;9(3). pii: E81. https://doi.org/10.3390/ diagnostics 9030081

5. Sakamoto H, Kitano M, Suetomi Y, Maekawa K, Takeyama Y, Kudo M. Utility of contrast-enhanced endoscopic ultrasonography for diagnosis of small pancreatic carcinomas. Ultrasound Med Biol. 2008;34(4):525-32. https://doi. org/10.1016/j.ultrasmedbio.2007.09.018

6. Eloubeidi MA, Chen VK, Eltoum IA, Jhala D, Chhieng DC, Jhala N, et al. Endoscopic ultrasound-guided fine needle aspiration biopsy of patients with suspected pancreatic cancer: diagnostic accuracy and acute and 30-day complications. Am J Gastroenterol. 2003;98(12):2663-8. https:// doi.org/10.1111/j.1572-0241.2003.08666.x

7. Brand B, Pfaff T, Binmoeller KF, Sriram PV, Fritscher-Ravens A, Knöfel WT, et al. Endoscopic ultrasound for differential diagnosis of focal pancreatic lesions, confirmed by surgery. Scand J Gastroenterol. 2000;35(11):1221-8. https://doi. org/10.1080/003655200750056736

8. Hewitt MJ, McPhail MJ, Possamai L, Dhar A, Vlavianos P, Monahan KJ. EUS-guided FNA for diagnosis of solid pancreatic neoplasms: a meta-analysis. Gastrointest Endosc. 2012;75(2):319-31. https://doi.org/10.1016/j.gie.2011.08.049

9. Zhang L, Sanagapalli S, Stoita A. Challenges in diagnosis of pancreatic cancer. World J Gastroenterol. 2018;24(19):20472060. https://doi.org/10.3748/wjg.v24.i19.2047

10. Itoi T, Tsuchiya T, Itokawa F, Sofuni A, Kurihara T, Tsuji $S$, et al. Histological diagnosis by EUS-guided fine-needle aspiration biopsy in pancreatic solid masses without on-site cytopathologist: a single-center experience. Dig Endosc. 2011;23 Suppl 1:34-8. https://doi.org/10.1111/j.14431661.2011.01142.x
11. Erturk SM, Mortelé KJ, Tuncali K, Saltzman JR, Lao R, Silverman SG. Fine-needle aspiration biopsy of solid pancreatic masses: comparison of CT and endoscopic sonography guidance. AJR Am J Roentgenol. 2006;187(6):1531-5. https://doi.org/10.2214/AJR.05.1657

12. Stella SF, Van Borsel M, Markose G, Nair SB. ImageGuided Percutaneous Biopsy for Pancreatic Lesions: 10-Year Experience in a Tertiary Cancer Center. Can Assoc Radiol J. 2019;70(2):199-203. https://doi.org/10.1016/j. carj.2018.10.014

13. Hébert-Magee S, Bae S, Varadarajulu S, Ramesh J, Frost AR, Eloubeidi MA, et al. The presence of a cytopathologist increases the diagnostic accuracy of endoscopic ultrasound-guided fine needle aspiration cytology for pancreatic adenocarcinoma: a meta-analysis. Cytopathology. 2013;24(3):159-71. https://doi.org/10.1111/cyt.12071

14. Holt BA, Varadarajulu S, Hébert-Magee S. High-quality endoscopic ultrasound-guided fine needle aspiration tissue acquisition. Adv Ther. 2014;31(7):696-707. https://doi. org/10.1007/s12325-014-0129-5

15. Gonçalves B, Soares JB, Bastos P. Endoscopic Ultrasound in the Diagnosis and Staging of Pancreatic Cancer. GE Port J Gastroenterol. 2015;22(4):161-171. https://doi. org/10.1016/j.jpge.2015.04.007

16. Low G, Panu A, Millo N, Leen E. Multimodality imaging of neoplastic and nonneoplastic solid lesions of the pancreas. Radiographics. 2011;31(4):993-1015. https://doi. org/10.1148/rg.314105731

17. Ayres LR, Kmiotek EK, Lam E, Telford JJ. A Comparison of Endoscopic Ultrasound-Guided Fine-Needle Aspiration and Fine-Needle Biopsy in the Diagnosis of Solid Pancreatic Lesions. Can J Gastroenterol Hepatol. 2018;2018:1415062. https://doi.org/10.1155/2018/1415062

18. Polkowski M, Jenssen C, Kaye P, Carrara S, Deprez P, Gines A, et al. Technical aspects of endoscopic ultrasound (EUS)guided sampling in gastroenterology: European Society of Gastrointestinal Endoscopy (ESGE) Technical GuidelineMarch 2017. Endoscopy. 2017;49(10):989-1006. https:// doi.org/10.1055/s-0043-119219

19. Kitano M, Yamashita Y. New Imaging Techniques for Endoscopic Ultrasonography: Contrast-Enhanced Endoscopic Ultrasonography. Gastrointest Endosc Clin $\mathrm{N}$ Am. 2017;27(4):569-583. https://doi.org/10.1016/j. giec.2017.06.002

20. Seicean A, Jinga M. Harmonic contrast-enhanced endoscopic ultrasound fine-needle aspiration: Fact or fiction? Endosc Ultrasound. 2017;6(1):31-36. https://doi. org/10.4103/2303-9027.196917 